\title{
RESET
}

Recherches en sciences sociales sur Internet

\section{Le jeûne d'internet}

Réduction et abstinence des médias numériques au service de l'expérience spirituelle

Internet fast. Reduction and abstinence from digital media and spiritual experience

Isabelle Jonveaux

\section{(2) OpenEdition}

Journals

Édition électronique

URL : https://journals.openedition.org/reset/2357

DOI : $10.4000 /$ reset.2357

ISSN : 2264-6221

Éditeur

Association Recherches en sciences sociales sur Internet

Référence électronique

Isabelle Jonveaux, « Le jeûne d'internet », RESET [En ligne], 9 | 2020, mis en ligne le 06 octobre 2020,

consulté le 21 mai 2021. URL : http://journals.openedition.org/reset/2357 ; DOI : https://doi.org/

$10.4000 /$ reset.2357

Ce document a été généré automatiquement le 21 mai 2021.

(c) Association Recherches en sciences sociales sur Internet 


\section{Le jeûne d'internet}

Réduction et abstinence des médias numériques au service de l'expérience spirituelle

Internet fast. Reduction and abstinence from digital media and spiritual experience

Isabelle Jonveaux

1 Alors qu'Internet est devenu un élément incontournable de pratiquement toutes les dimensions de la vie quotidienne, y compris pour la vie spirituelle, des tendances à la déconnexion prennent de plus en plus de place dans le paysage des pratiques liées aux médias numériques. L'apparition de différentes formes de stages de déconnexion montre qu'ils répondent à une demande certaine. Parallèlement, le droit à la déconnexion pour les salariés a même été inscrit dans le code du travail le $1^{\mathrm{er}}$ janvier 2017. Ainsi que le remarquaient déjà en 2010 Kellner, Massou et Morelli,

« dans la littérature scientifique - francophone ou anglo-saxonne - la question des non-usages est rarement posée comme une problématique en tant que telle. Elle y est abordée comme un cas particulier d'usage si ce n'est, la plupart du temps, comme un défaut d'usage. Or, la question du non-usage est suffisamment complexe pour justifier qu'on la traite en tant que telle. » (Kellner, Massou, Morelli, 2009: 8)

2 L'augmentation des tendances de déconnexion nécessite donc que l'on s'y intéresse comme des pratiques en soi.

3 Les motivations qui conduisent à la déconnexion sont variées, notamment dans le monde professionnel, mais je m'intéresserai ici exclusivement à la limitation de l'utilisation d'Internet ou abstinence pour des motifs spirituels. Cette déconnexion prend place dans différents contextes, de religion institutionnelle ou de nouvelle spiritualité. Pour le sociologue allemand Hubert Knoblauch, les spiritualités «évitent les organisations ecclésiales strictes, elles sont orientées vers une approche globale et mettent un accent important sur les expériences subjectives de la transcendance " (Knoblauch, 2009: 41). Nous considérerons ici comme religion institutionnelle, reprenant la définition de Danièle Hervieu-Léger, un « dispositif idéologique, pratique et symbolique, par lequel est constituée, entretenue, développée et contrôlée la conscience (individuelle et collective) de l'appartenance à une lignée croyante 
particulière » (Hervieu-Léger, 1993 : 119). Le jeûne d'Internet à but spirituel prend place au sein de mutations du système ascétique traditionnel où les pratiques de jeûne alimentaire comme prescription de l'Eglise institutionnelle semblent tomber en désuétude. En revanche, les jeûnes librement consentis par l'individu, sous la forme et lors de la période qu'il souhaite, fleurissent alors même qu'ils sont souvent plus stricts que les anciens jeûnes religieux. Cela renvoie à la distinction effectuée par Paul Heelas et Linda Woodhead entre « life-as » et " subjective life »:

"Les formes de 'vie en tant que' (life-as) du sacré qui mettent l'accent sur une source transcendantale de signification et d'autorité à laquelle les individus doivent se conformer au détriment de la cultivation leurs vies subjectives uniques, ont tendance à être en déclin.

Les formes de vie subjective (subjective-life) qui mettent l'accent sur les sources intérieures de signification et d'autorité ainsi que sur la cultivation de la sacralisation des vies subjectives uniques, ont tendance à être en augmentation. » (Heelas, Woodhead, $2005: 6$ )

4 La frontière n'est toutefois pas hermétique car les pratiques de spiritualité globale ou de « vie prise dans sa globalité » (Höllinger, Tripold, 2012 : 15), corps, âme, esprit, sont aussi actuellement vécues par des croyants catholiques en recherche de réincarnation de leur vie religieuse.

Dans quel but les individus limitent-ils leur accès à Internet? En quoi pensent-ils que le jeûne peut être bénéfique pour leur vie spirituelle? Inversement, dans quelle mesure considèrent-ils que l'usage des médias numériques peut être nocif pour la vie religieuse ? Cette limitation ou renoncement temporaire aux médias numériques estelle recommandée ou exigée par des autorités religieuses ou renvoie-t-elle à une démarche personnelle de l'individu ? La question de la religion et Internet ne concerne pas seulement la pratique religieuse en ligne ou ce que nous pouvons appeler avec Christopher Helland la online religion qui se vit «sur et à travers le media Internet " (Helland, $2005: 12$ ), mais aussi la façon dont l'usage des médias numériques est intégré dans la pratique religieuse, ce qui suppose d'éventuels temps d'abstinence, de limitation, en un mot, une discipline. Etudier la déconnexion permet donc de repenser la catégorie d'ascèse, concept classique en sociologie des religions, dans ce concept de la pratique en ligne et de l'intégration des médias dans la vie spirituelle.

6 Cet article vise à étudier un rapport particulier aux médias dans leur non-usage, et s'intéresse donc principalement à des pratiques développées hors-ligne par les acteurs. Il en découle que les données de terrain proviennent essentiellement d'entretiens et d'observations hors-ligne. Après avoir présenté les différentes formes de déconnexion, j'expliquerai comment cette dernière prend place sur trois types de terrains étudiés, que sont la vie monastique catholique, les catholiques pratiquants laïcs et enfin, les jeûneurs qui effectuent des stages de jeûne à but spirituel sans se rattacher à une religion institutionnelle précise. Finalement, la dernière partie s'intéressera aux buts recherchés dans la déconnexion pour comprendre notamment en quoi elle peut servir un but spirituel et s'apparenter à une nouvelle forme d'ascèse.

7 Présentation des terrains étudiés: Cet article repose sur les données de plusieurs recherches menées successivement et parallèlement. La première concerne le milieu monastique catholique, identifié par Max Weber comme lieu de la virtuosité ascétique (Weber, 1996: 262). La seconde porte sur les recompositions et transferts de l'ascèse contemporaine dans la société séculière, notamment dans le cadre des semaines de jeûne. Enfin, le troisième type de recherches mobilisé renvoie à l'étude de l'utilisation 
des médias numériques par les croyants catholiques. Ces terrains présentent deux niveaux de recoupement: l'ascèse et les pratiques numériques, avec une question commune concernant la limitation de l'utilisation d'Internet pour des motifs religieux. D'où l'intérêt de synthétiser dans cet article ces différentes tendances à la déconnexion pour des motifs religieux ou spirituels.

Les enquêtes en monastères ici mobilisées ont été menées essentiellement depuis 2007, sous l'angle de l'usage d'Internet dans la vie monastique puis des mutations actuelles de l'ascèse ${ }^{1}$. J'ai étudié des communautés dans sept pays d'Europe, cinq d'Afrique et l'Argentine mais par souci d'unité géographique, je ferai appel ici aux données concernant la France et l'Autriche. Ma recherche d'habilitation ${ }^{2}$ a porté sur de nouvelles pratiques de jeûne intégral à but spirituel, reposant sur une approche globale " corps-âme-esprit ». 45 entretiens semi-directifs ont été menés avec des jeûneurs qui pratiquent des jeûnes selon la méthode Buchinger-Lützner qui consiste à ne prendre que des liquides pendant une semaine ou plus, bannissant toute alimentation solide et centrant cette démarche sur l'idée d'une purification à trois niveaux, du corps, de l'âme et de l'esprit. Une observation participante a notamment été menée sur une semaine de jeûne et randonnée en Autriche en 2014 et sur une semaine de formation des entraîneurs de jeûne en Autriche en 2016. Je m'appuierai aussi sur les réponses apportées à un questionnaire diffusé d'une part allemand dans un groupe Facebook autrichien de jeûne Buchinger ${ }^{3}$ et d'autre part en français, par mail, pour des participants à des semaines de jeûne Buchinger organisés par une association française dans des monastères ${ }^{4}$. Le questionnaire a été envoyé par l'association aux anciens participants. J'utiliserai essentiellement les réponses apportées à la question ouverte : "Vous est-il important de réduire votre utilisation des médias (téléphone, Internet) pendant votre session de jeûne? Si oui, pourquoi?». Les questions étant similaires dans les deux questionnaires, je synthétiserai ici les réponses car les questionnaires ont apporté beaucoup moins de réponses que ce qui était attendu : 65 pour le questionnaire en allemand, 83 pour celui en français.

Enfin, j'aurai aussi recours à deux autres questionnaires portant sur l'utilisation des médias numériques dans le milieu catholique. Le premier mené en 2014 auprès de 292 jeunes catholiques autrichiens avait pour but d'étudier la manière dont ils rendent visible leur affiliation religieuse sur les réseaux sociaux numériques. Il a été diffusé dans des groupes Facebook s'adressant à des jeunes catholiques. Le second a été diffusé en 2017 auprès d'utilisateurs des offres en ligne de la pastorale des jeunes organisée par les Franciscains en Autriche. Le questionnaire a récolté 101 réponses.

\section{Les formes de la déconnexion}

10 Les démarches de déconnexion ici étudiées sont des formes de renoncement volontaire aux médias numériques dont il faut préciser ici les modalités.

\section{Le non-usage d'internet}

11 Le jeûne d'internet pour motifs spirituels se rapporte à une catégorie spécifique de non-usage de l'internet qui prend place dans un contexte où l'accès aux médias est possible. Si pour quelque raison que ce soit (coût, barrière sociale, manque de réseau), l'accès n'est pas possible, on ne peut pas parler de renoncement volontaire. 
"Sally Wyatt, Graham Thomas et Tiziana Terranova (2002) identifient quatre groupes de non-utilisateurs: les "abandonnistes volontaires" (rejecters) qui n'utilisent plus l'internet par choix personnels ; les "abandonnistes involontaires" (expelled) qui ont arrêté d'utiliser pour des raisons qui ne relèvent pas cette fois de leur volonté; les "exclus" (excluded) qui ne peuvent avoir d'accès par manque d'infrastructure ou de moyens socio-économiques et les "résistants" (resisters) qui n'ont jamais utilisé l'internet par choix. » (Granjon, $2010: 41$ )

Dans le cas présent, il s'agit d'une sous-catégorie spécifique d'abandonnistes volontaires qui le font par choix personnel et pour une période déterminée. Ce n'est pas, en règle générale, un abandon complet et définitif de l'usage des nouvelles technologies de communication. En miroir de la catégorie d'utilisateurs occasionnels élaborée par Levingstone et Helpser (cité par Granjon, 2010:42), nous pouvons construire la catégorie d'abandonnistes occasionnels volontaires. Toutefois, il s'agit ici d'un non-usage volontaire qui ne résulte pas initialement « d'un manque d'intérêt dans les gratifications qu'on pourrait tirer de l'innovation » (Martin, von Pape, 2010 : 136) comme Corinne Martin et Thelo von Pape l'ont identifié dans le cadre du téléphone portable auprès d'étudiants et d'adolescents. L'intérêt et l'envie sont présents mais les acteurs décident de suspendre leur usage pour répondre à d'autres motivations, en l'occurrence ici, spirituelles. D'où la difficulté qu'ils rencontrent parfois à tenir cette décision comme nous allons le voir.

Loin de la nécessité non choisie de réduire ou de suspendre l'usage de l'internet, ces acteurs sont capables d'élaborer le discours justifiant leur déconnexion. La justification serait en effet une caractéristique de l'ascèse selon Aviad Kleinberg : "Quels que soient les mobiles personnels de l'ascète, il est en mesure de faire valoir, par ses actes ou par ses paroles, qu'il se plie à une tradition ascétique existante (fût-elle marginale) et que ce ne sont pas là des errements ou des caprices personnels. ( (Kleinberg, 2005 : 114) La capacité de justifier le non-usage "Dans la très grande majorité des cas, les usages restreints sont choisis et justifiés selon une rationalité que les personnes interrogées sont capables d'expliquer. ( (Kellner, Massou, Morelli 2010 : 15)

La déconnexion n'est pas nécessairement une démarche religieuse ou spirituelle comme le montrent les offres de plus en plus nombreuses de stages de "désintoxication des médias ». Partant du constat des formes de dépendance voire d'addiction que peuvent susciter les médias numériques, notamment le smartphone, se développe un marché de séjours de déconnexion. Par exemple, l'entreprise française « Into the tribe » créée en 2015 propose des stages dans différents pays d'Europe où une application est installée en début de séjour pour bloquer toutes les fonctions du smartphone hormis les appels. La proposition "Digitale Detox Retreat auf Kreta " émanant d'une agence de voyages suisse utilise quant à elle l'expression de jeûne digital : «S'accorder un jeûne digital, un temps de pause digital ou une pure abstinence digitale est une bonne possibilité pour rester productif et équilibré dans notre monde fortement connecté5 ». Le vocabulaire ici utilisé est bien celui de la retraite spirituelle ascétique. Le concept d'abstinence renvoie directement au monde de l'ascèse religieuse. Même si le but premier n'est pas explicitement spirituel, ces sociétés utilisent la rhétorique traditionnelle de l'ascèse pour vendre leurs séjours. 


\section{Comment une consommation devient ascèse}

$\mathrm{Au}$ regard de la multitude des consommations possibles pour les individus contemporains, l'on peut s'interroger sur la raison qui pousse à inclure les usages d'Internet dans les pratiques ascétiques. Les tendances à la déconnexion s'appuient toutes sur un bilan négatif de la surconsommation d'Internet qui entraîne des formes de dépendance, d'où la nécessité d'une démarche consciente pour s'en abstenir. En effet, l'enquête du « Baromètre du numérique » 2016 relevait en France :

«Pour un internaute sur deux, il est impossible de se passer d'internet plus de quelques jours ( +9 points en cinq ans). Comme en 2011, un quart pensent pouvoir se passer d'internet pendant une ou deux semaines. Mais, alors que $36 \%$ des internautes pouvaient s'en passer plus de 15 jours en 2011, ils ne sont plus que $27 \%$ cette année. Les internautes sont donc à la fois plus nombreux et plus « dépendants " de leur accès à internet. ${ }^{6}$

Dans le questionnaire sur le jeûne, une femme de 56 ans explique en effet réduire sa consommation de médias numériques pendant le jeûne comme "désintoxication mentale », ce que l'on retrouve dans le questionnaire en allemand sous la forme de «mediale Entgiftung» (détoxication médiale) (Femme, 50 ans), et une autre de 59 ans parle de se rendre « libre du virtuel ». Dans ces réponses transparaissent en effet l'idée d'une dépendance dont il faut se délivrer. Une femme de 39 ans parle de " jeûner aussi des esclavages modernes ». Les tendances à la déconnexion dans une démarche de réduction volontaire ou d'abstinence prennent donc place dans un contexte où non seulement l'usage des médias numériques est suffisamment répandu pour que son utilisation ne constitue plus une forme de distinction, mais aussi dans la prise de conscience d'effets négatifs qui sont encore mal maitrisés. Inversement en effet, les enquêtés mettent en évidence dans les justifications que la déconnexion « leur fait du bien " (étudiante de 24 ans), qu'elle leur permet de mieux rencontrer les autres mais aussi de retrouver une qualité de sommeil lors qu'ils renoncent à utiliser leur téléphoner dans leur lit ou juste avant d'aller dormir.

\section{Le jeûne des médias}

17 Parler ici de jeûne d'internet renvoie à une image qui touche pour une part à la nourriture et pour une autre part, au monde religieux ascétique. S'agit-il concernant les médias numériques d'une métaphore ou d'un sens premier ? Jeûner signifie en premier lieu, et selon la définition du Larousse, "s'abstenir de manger » (2019). Le jeûne est dans la spiritualité chrétienne le premier pas de toute ascèse car il cherche à dompter ce qui serait le plus grand vice, celui de la gourmandise. Michel Foucault y voit une « chaîne causale » du péché :

«Au principe des autres, le couple gourmandise-fornication, comme « un arbre géant qui étend au loin son ombre ", doit être déraciné. De là l'importance ascétique du jeûne comme moyen de vaincre la gourmandise et de couper court à la fornication. Là est la base de l'exercice ascétique, car là est le commencement de la chaîne causale » (Foucault, 1994 : 297)

Le but du jeûne est aussi, comme le souligne Assouly, « de réaffirmer la prédominance de l'âme en limitant l'augmentation de la tempérance du sang et ses conséquences funestes. » $(2002: 140)$. 
19 Le jeûne consiste à se priver de nourriture selon différentes modalités, que ce soit de manière quantitative (manger moins ou pas du tout) ou qualitative (s'abstenir de certains aliments comme la viande), et sur des périodes définies plus ou moins longues (une semaine, 40 jours du carême) ou de manière régulière et récurrente (chaque vendredi par exemple). Utiliser le terme de jeûne concernant les médias numériques renvoie donc dans l'imaginaire collectif chrétien à ce pilier de l'ascèse. Dans les entretiens avec des jeûneurs des sessions Buchinger, le terme de jeûne a été employé uniquement concernant l'alimentation et les médias. Par exemple, une étudiante autrichienne de 24 ans à ma question de savoir sous quelle forme elle jeûne, explique à la suite et sans distinction avoir jeûné de chocolat, de sucre, de viande et avoir essayé de jeûner de téléphone portable. Et un séminariste qui a suivi une semaine de jeûne Buchinger explique ainsi en quoi le jeûne a été une belle expérience pour lui :

«Ce n'était pas en premier lieu la nourriture, ce n'était pas le plus difficile, mais simplement, que l'on essaie pendant cette semaine de réduire tous les travaux, les activités. Par exemple, que l'on ne doit pas obligatoirement regarder la télévision, que je ne dois pas checker mes mails toutes les deux heures, que je puisse laisser mon portable dans ma chambre.» (02.2015)

Cela signifie que l'usage des médias digitaux est mis sur le même plan de besoin primaire que l'alimentation. Le jeûne signifie alors maîtrise de la consommation de manière générale, et surtout des consommations dont la surabondance produit des effets nocifs.

Selon Max Weber, l'ascèse est « une approche méthodique de l'homme tout entier » et imprime un « ordre [...] dans la conduite de vie » (Weber, $2002: 195)$ qui concerne tous les aspects de l'existence dont le rapport aux médias, mais aussi à la cigarette bannie des semaines de jeûne par exemple. Se priver d'Internet ou de téléphone portable a donc pour fonction de travailler à cet « ordre » dans la conduite de vie. Il s'agit en effet pour certains jeûneurs de modifier leurs habitudes en profondeur pendant la période intensive de jeûne afin de conserver ensuite certaines habitudes nouvelles et travailler ainsi sur soi :

«L'accent est mis sur le rapport à soi qui permet de ne pas se laisser emporter par les appétits et les plaisirs, de garder vis-à-vis d'eux maîtrise et supériorité, de maintenir ses sens dans un état de tranquillité, de demeurer libre de tout esclavage intérieur à l'égard des passions, et d'atteindre à un mode d'être qui peut être défini par la pleine jouissance de soi-même ou la parfaite souveraineté de soi sur soi. » (Foucault, 1983 : en ligne)

C'est par exemple le but du programme d'abstinence «Exodus $90^{7}$ », de modifier en 90 jours les habitudes qui ne servent pas de but spirituel, notamment dans l'utilisation d'Internet. Ce programme dit se baser sur des résultats scientifiques qui montrent que le cerveau d'un homme peut être modifié - « et retourner à un état plus naturel » - sur une période de 90 jours. Ce serait le temps nécessaire pour se libérer de mauvaises habitudes, notamment celles liées à l'usage des médias digitaux. De même, un jeûneur autrichien explique comment il a voulu modifier volontairement son comportement vis-à-vis d'Internet pendant ses périodes de jeûne :

«J'ai jeûné, là par exemple l'année dernière, de ne pas regarder de série avant d'aller dormir. J'avais cet habitus de toujours regarder quelque chose, un documentaire ou une vidéo sur YouTube et de m'endormir là-dessus. Mais consciemment de ne pas le faire et dire une prière et d'aller au lit. [...] Que l'on trouve la place juste pour la technique. Qu'elle n'ait pas de place fixe dans la vie, ou 
pas de place absolue, si on peut dire.» (04.2016, homme, 22 ans, étudiant en théologie)

$$
\text { qui peuvent autant concerner l'alimentation que l'usage des médias ou toute autre }
$$
consommation.

\section{L'ascèse des virtuoses et des masses}

\section{Des moines et moniales catholiques}

L'ascèse est en premier lieu selon Weber le domaine des virtuoses. Ces derniers sont ceux qui se consacrent entièrement à la recherche du salut. On observe en effet que certains groupes radicaux comme les Juifs ultra-orthodoxes (Haredim) limitent consciemment leur utilisation d'Internet. Ainsi en 2007, seulement $23 \%$ des juifs Haredim en Israël avaient une connexion Internet contre $92 \%$ des juifs séculiers (Cohen, 2013 : 46). Chez les Haredim, le refus est justifié par des raisons religieuses et explicitement anti-modernes. On se trouve ici dans le cas d'une ascèse consciente ou en l'occurrence un refus de la modernité comme potentiellement pernicieuse pour la religiosité. Kellner, Massou et Morelli identifient en effet le refus explicite de la modernité comme motivation de non-usage : «[...] être non-usager témoigne parfois d'un positionnement (refus de la modernité et de la suprématie des technologies, résistance-s au changement...) qui relève d'un choix délibéré, affirmé ou revendiqué (position de principe)» (Kellner, Massou, Morelli 2010: 8). De manière générale, le repos du shabbat est aussi observé dans une réduction de l'usage d'Internet comme de toute autre technique. Dans le cas des Haredim, certains sites Internet sont même fermés pendant le shabbat:

«Par exemple, si vous accédez aux pages du parti politique israélien Haredim, le Shas, depuis la République Tchèque pendant le temps du Sabbat (en République Tchèque), vous verrez apparaitre la notice suivante : 'Service Sabbat du web : Chers surfers! Ce site observe le Sabbat et les jours fériés juifs, ces jours-là, les surfers ne peuvent pas entrer. En ce moment, le site est fermé parce que : aujourd'hui est le jour du Sabbat [ou jour de congé juif ]. Le Sabbat en République Tchèque finira dans $\mathrm{X}$ heurs et $\mathrm{Y}$ minutes. Nous serons ravis de vous rendre service un autre jour de la semaine. » (Čejka, 2009 :7-8, notre trad.).

Dans le monde catholique, la virtuosité religieuse est portée selon Weber essentiellement par les moines et moniales. Ces derniers élaborent aussi une discipline concernant l'usage des technologies de l'information et de la communication (Jonveaux, 2013). Cela ne signifie pas qu'ils refusent toute utilisation de l'Internet par refus de la modernité - ils savent au contraire les mettre à profit autant pour des buts spirituels (pastorale) qu'économiques -, mais ils tâchent d'y imprimer une discipline pour limiter son usage afin de l'intégrer dans la vie monastique. Seules certaines communautés féminines à clôture stricte et dont la moyenne d'âge est élevée pratiquent un réel refus de l'Internet. Quelques-unes ne possèdent encore qu'une adresse mail pour toute la communauté, comme j'ai pu le voir en 2015 en Argentine ou en 2019 au Bénin. D'autres, notamment en France, reconnaissent l'importance de l'accès personnel à Internet mais ont installé une salle informatique pour éviter d'avoir une connexion dans les chambres individuelles, ce qui permet d'établir un contrôle social diffus. De nombreux exemples font aussi état d'une coupure de la connexion entre le dernier office du soir et le premier office du matin pour respecter « le grand 
silence de la nuit » de la règle bénédictine et éviter que les moines passent leur nuit sur Internet. Par exemple, l'abbé d'un monastère français explique :

« On n'a pas [accès à Internet] de la cellule. De même, on a cette discipline, et j'y tiens beaucoup, on n'a pas d'ordinateur dans la cellule. C'est notre limite. On s'est donné une limite pour l'Internet entre $20 \mathrm{~h} 30$ le soir et $8 \mathrm{~h}$ le matin. Il y a un système, on coupe le réseau. Et ça, c'est aussi une ascèse. Elle n'est pas physique ou corporelle, mais elle est bien réelle. On bute assez facilement sur la limite. Sur le moment, on râle, et après on n'y pense plus. Même moi, ça m'arrive, le soir de me dire il faudrait que je fasse partir ce mail et puis, ha oui mince, j'ai coupé le réseau. (Rire) Je pense que c'est important qu'on ait ce genre de limites. » (04.2011, abbé)

Les communautés autrichiennes de spiritualité bénédictine qui sont, du fait de leur histoire, beaucoup plus actives à l'extérieur, ont rarement ce genre de discipline communautaire, mais il est courant que les moines et moniales individuels s'imposent une discipline personnelle (Jonveaux, $2018: 105)$. Un bénédictin disait par exemple avoir choisi de ne pas avoir d'ordinateur dans sa cellule. Certains la mettent directement en rapport avec le jeûne monastique. Ainsi dans une communauté cistercienne proche de Vienne, des moines affirment ne pas utiliser Internet les jours de jeûne de la communauté. L'un d'eux - qui a par ailleurs 5000 amis sur Facebook - dit ne pas aller sur Internet les mercredi et vendredi, jours de jeûne prévus par la règle de saint Benoît quand un autre s'en abstient aussi le lundi, jour moindre de jeûne. Les jours de jeûne monastique sont traditionnellement marqués par l'ascèse alimentaire d'abstinence de viande. Le jeûne monastique devient alors aussi un jeûne des médias numériques.

\section{Des croyants institutionnels}

Le jeûne de l'internet apparaît chez les croyants comme accompagnant des périodes de plus forte intensité religieuse ou les périodes traditionnelles de jeûne recommandées par l'institution religieuse. Ainsi observe-t-on chez les catholiques pratiquants des pratiques de retrait des réseaux sociaux en ligne et de réduction de la consommation d'Internet pendant le carême. Le questionnaire mené en 2014 auprès de jeunes catholiques autrichiens a montré qu'un tiers des catholiques de cet échantillon qui vont à la messe une fois par semaine ou plus trouvent important de réduire leur consommation des réseaux sociaux dans les moments forts du temps religieux comme le Carême contre $12 \%$ de l'échantillon total.

Tableau : Importance de limiter l'utilisation des réseaux sociaux numériques en fonction de la fréquentation de la messe (2014)

\begin{tabular}{|l|l|l|l|l|l|l|}
\hline Fréquentation messe & $\begin{array}{l}\text { Très } \\
\text { important }\end{array}$ & Important & $\begin{array}{l}\text { Pas vraiment } \\
\text { important }\end{array}$ & $\begin{array}{l}\text { Pas } \\
\text { important }\end{array}$ & $\begin{array}{l}\text { Pas du tout } \\
\text { important }\end{array}$ & Total \\
\hline $\begin{array}{l}\text { Une fois par semaine } \\
\text { ou plus }\end{array}$ & $11,50 \%$ & $19,20 \%$ & $57,70 \%$ & $7,70 \%$ & $3,90 \%$ & $100 \%$ \\
\hline $\begin{array}{l}\text { Au moins une fois par } \\
\text { mois }\end{array}$ & $0 \%$ & $25 \%$ & $36,10 \%$ & $33,30 \%$ & $5,60 \%$ & $100 \%$ \\
\hline Quelques fois par an & $2,30 \%$ & $13,60 \%$ & $40,90 \%$ & $34,10 \%$ & $9,10 \%$ & $100 \%$ \\
\hline
\end{tabular}




\begin{tabular}{|l|l|l|l|l|l|l|}
\hline $\begin{array}{l}\text { Seulement pour les } \\
\text { grandes fêtes } \\
\text { religieuses }\end{array}$ & $0 \%$ & $9,10 \%$ & $47,30 \%$ & $32,70 \%$ & $10,90 \%$ & $100 \%$ \\
\hline Jamais ou presque & $0 \%$ & $1,10 \%$ & $14,60 \%$ & $71,90 \%$ & $12,40 \%$ & $100 \%$ \\
\hline $\begin{array}{l}\text { Total \% } \\
\text { N=250 }\end{array}$ & $1,6 \%$ & $10,4 \%$ & $34 \%$ & $44,4 \%$ & $9,6 \%$ & $100 \%$ \\
\hline Ensemble & 4 & 26 & 85 & 111 & 24 & 250 \\
\hline
\end{tabular}

La déconnexion pour motifs religieux augmente donc avec la pratique. Dans le questionnaire concernant la pastorale des jeunes des Franciscains en Autriche, 40,8\% a répondu trouver très important de se déconnecter lors des moments importants pour la foi et 28,6 \% plutôt important. Concernant les moments de la déconnexion, le carême arrive en première place $(57,5 \%)$ et les retraites spirituelles juste après $(56,3 \%)$. Les temps de prière et la liturgie sont aussi fréquemment cités comme moments de déconnexion, ce qui implique que d'éteindre ou ne pas regarder son téléphone portable pendant un temps de prière ou la messe est une démarche consciente de déconnexion de la part des enquêtés.

Sur les réseaux sociaux, ce retrait est annoncé avec des photos de profil ou un message annonçant l'absence pour la durée déterminée. Il existe ainsi des groupes tels que " Je n'irai pas sur Facebook les vendredis de Carême » et des images «Facebook Fasten " (jeûne d'Internet en allemand) ou "Absent de Facebook pendant le carême » en photo de profil. En Autriche, la pastorale des jeunes catholique (Katholische Jugend) propose depuis le carême 2016 de mettre une couleur unie (vert clair ou bleu violet) comme photo de profil ou de couverture comme "virtuelles Fastentuch » (voile de carême virtuel), faisant ainsi écho aux tissus violets qui couvrent les peintures d'autel et les statues dans les églises pendant tout le temps du carême. L'un de mes contacts, une fille de 22 ans en 2016, avait mis en photo de profil une photo de désert avec l'inscription : «Carême : Prière, Pénitence, Partage ".

Dans la démarche de jeûne "Exodus» précédemment évoquée, il est précisé que l'utilisation des médias numériques doit être fortement réduite : "Computer for research ONLY ». L'une des raisons est notamment que la chasteté est une valeur centrale de ce programme et que l'Internet est considéré comme le vecteur principal de diffusion de la pornographie. La limitation volontaire de l'usage d'Internet peut donc accompagner des périodes traditionnelles de jeûne pour réintensifier la vie spirituelle, même si les acteurs ne jeûnent pas nécessairement du point de vue alimentaire. Il peut aussi être une pratique qui vise à remplacer le jeûne alimentaire quand ce dernier a perdu du sens pour les croyants.

31 Ces exemples montrent la manière dont les croyants intègrent l'usage des médias numériques dans leur comportement et leur pratique religieux, ce qui aboutit à des temps d'abstinence ou de limitation. Cette dimension de l'intégration de la consommation des médias numériques dans le comportement religieux voire l'ascèse se retrouve notamment dans la corrélation négative qui a été mise en lumière entre pratique religieuse et usage de la pornographie sur Internet (Short, Kasper, Wetterneck, 2015). De la même manière, on observe dans le milieu musulman la mise 
en scène par des Youtubeuses de leur pudeur comme expression de la morale musulmane (Le Guen-Fromenti, 2015).

\section{Les jeûneurs de spiritualité globale} récurrent des entretiens menés avec des organisateurs ou des participants à des semaines de jeûne selon la méthode Buchinger-Lützner. Cette pratique de jeûne s'inscrit dans le cadre de ce que l'on peut appeler la spiritualité globale ou holiste visant une harmonie du corps, de l'âme et de l'esprit (Höllinger, Tripold, 2012). Les semaines de jeûne étudiées ne s'inscrivent pas dans une religion institutionnelle donnée mais s'affirment comme spirituelles. Toutefois, ce jeûne peut aussi être intégré dans la pratique catholique comme nous allons le voir.

Labstinence des médias est un élément recommandé par les organisateurs des stages de jeûne, comme observé dans le cadre de l'enquête sur une semaine de jeûne et randonnée.

ans les entretiens, le jeûne des médias a souvent été spontanément évoqué sans question préalable de ma part. Par exemple, un séminariste de Graz, racontant sa semaine de jeûne Buchinger effectuée avec le séminaire comme retraite d'Avent, cite l'abstinence de télévision, téléphone portable et courriers électroniques en deuxième position après la nourriture. Le jeûne des médias n'est pas présenté comme un élément surajouté à la démarche de jeûne alimentaire, mais une part du jeûne en soi puisque celui-ci peut être compris comme englobant des domaines plus vastes. Ainsi un enquêté disait qu'il s'agit de « jeûner en tout ». Dans le questionnaire, à la question de savoir s'il leur est important de réduire l'usage des médias pendant une semaine de jeûne, une femme de 70 ans répond : "Se polluer pendant le jeûne est un non-sens ». Une autre de 53 ans parle d'" une retraite également technologique». Tandis qu'un jeûneur autrichien explique :

"J'ai remarqué que tout ce qui me coûte un peu d'énergie, par exemple, le téléphone ou l'ordinateur, je l'ai complètement laissé de côté comme tout ce qui me déconcentre. Là j'essaie de me remettre tout de suite à méditer, ou de sortir, où ça me fait du bien, aussi ne pas rester trop longtemps à l'intérieur, mais sortir de temps en temps, justement ce qui me fait du bien. Et justement en aucun cas, le téléphone, l'ordinateur, regarder la télévision je ne le fais de toute façon pas, l'ordinateur normalement non plus. Ça doit être une atmosphère agréable tout le temps. » (04.2015, homme, 22 ans, étudiant)

L'abstinence des médias est aussi présentée par les jeûneurs comme un moyen de profiter au mieux des effets du jeûne alimentaire et de la démarche de purification en général. Par exemple, une organisatrice de stages de jeûne en Autriche explique :

«Dans mes groupes, je dis toujours ça, il n'y a de toute façon pas de portable, pas de télévision. Pendant qu'on fait les cataplasmes pour le foie, ils ne doivent pas lire, regarder la télévision ou utiliser leur portable. Ils ne doivent rien faire, c'est une chose importante pour que le cataplasme pour le foie fasse vraiment de l'effet, qu'on se laisse aller et qu'on retrouve le calme. Ces smartphones aujourd'hui sont ce qu'il y a de plus terrible, c'est une dépendance aujourd'hui. » (01.2017, femme, 50 ans, coach de jeûne).

Dans la même idée, une femme de 70 ans explique dans le questionnaire qu'il s'agit pour elle d'« être pleinement attentive au jeûne sans avoir l'esprit occupé par autre chose qui empêche d'être pleinement dans le jeûne ", quand une autre de 68 ans dit 
qu'elle jeûne des médias "pour laisser le travail se faire ». Dans ce cas, le jeûne des médias favorise les résultats attendus du jeûne alimentaire.

Le jeûne des médias se décline selon différentes modalités qui concernent un média ou des éléments du média concerné. Il n'existe donc pas une opposition binaire entre l'usage et le non-usage qui supposerait dans le cas du jeûne une abstinence totale, mais bien, comme l'ont remarqué Sonia Livingstone et Ellen Helsper $(2007: 682)$ concernant l'usage et le non-usage en général, un continuum selon une gradation variée des niveaux d'intensité et des motifs d'usage et non-usage. Comme je l'ai évoqué plus haut pour un stage de déconnexion, toutes les fonctions du smartphone sont bloquées sauf les appels téléphoniques. La réduction ou abstinence des médias peut tout d'abord consister en une abstinence totale de tout média, allant des journaux à l'ordinateur en passant par la télévision et le téléphone portable. Ce profil se retrouve notamment chez les individus de 50 ans et plus et les virtuoses religieux. Chez les plus jeunes, la réduction concerne le plus souvent certaines fonctions spécifiques des médias numériques comme les vidéos et séries ou les réseaux sociaux numériques. Le smartphone est alors le plus souvent cité. Le jeûne des médias numériques se décline donc sous différentes formes qui renvoient initialement aux usages habituels des individus. Le jeûne de séries en ligne ne concerne par exemple que ceux qui regardent ces séries. Tout comme observé dans le cas du jeûne alimentaire partiel où les individus sélectionnent les aliments auxquels il leur coûte le plus de renoncer - par exemple un moine qui disait jeûner en plus de chocolat pendant le carême, car là se trouvait la véritable ascèse pour lui - le jeûne des médias se construit en miroir des usages habituels.

\section{Pourquoi jeûner des médias}

\section{L'ascèse négative : s'abstenir de consommations nocives}

Le premier aspect du jeûne des médias que l'on retrouve pour le jeûne alimentaire est celui de la restriction par l'abstinence, donc le retrait. Otto Zöckler, dans sa typologie de l'ascèse distingue deux dimensions de l'ascèse individuelle, l'une négative " dans les actes de renoncement au monde et d'abstention de besoins vitaux (plus ou moins nécessaires) " (Zöckler, 1897: 5) et l'autre positive " dans les actes d'occupation (Beschäftigung) avec les choses divines ou, en un mot, l'élévation vers Dieu » (Zöckler $1897:$ 6) qui peut aussi s'appeler " ascèse spirituelle », et dont « le but est de s'élever des sens à l'esprit, du terrestre à Dieu, du monde au créateur " (id: 7). Le jeûne des médias numériques présente cette dimension négative dans les justifications d'abstinence ou de restriction par les effets négatifs de leur usage, y compris les conséquences sur la santé psychique et le sommeil. Le jeûne des médias pendant une semaine de jeûne alimentaire peut aussi avoir des dimensions bénéfiques sur la santé comme celles recherchées par l'abstinence de nourriture. Une jeûneuse autrichienne de 24 ans, explique par exemple :

« Oui, j'ai fait attention à ne pas trop regarder mon téléphone... Sinon, quand je suis trop fatiguée avant d'aller dormir ou quand c'est trop fatigant de lire un livre, je prends toujours mon téléphone [...]. Et là j'ai décidé de ne regarder Internet et Facebook que sur mon ordinateur. Quand j'éteins l'ordinateur, je laisse tout ça. Cette année, j'ai vraiment décidé de faire ça pendant tout mon temps de jeûne et j'ai réussi à le suivre. Que je laisse vraiment mon portable de côté quand je vais au lit 
[...]. Et j'ai remarqué que ça me fait du bien. S'endormir sans fixer mon portable. Et maintenant, je continue à le faire! (rire) C'était vraiment directement pendant le carême et ma semaine de jeûne, mais je continue à le faire.» (05.2016, femme, 25 ans, étudiante)

Les jeûneurs assimilent souvent leur abstinence des médias numériques à un refus de la société de (sur)consommation. Par exemple, dans le questionnaire, une femme de 60 ans écrit: "Me soustraire à la machine infernale de la surconsommation» ou un homme de 55 ans : « Fuir la société de consommation et montrer que ce n'est pas ça qui amène au bonheur ».

\section{Le nouveau désert}

Se déconnecter équivaut aujourd'hui à se retirer du monde et d'une grande partie des interactions sociales. En cela, la déconnexion rappelle le départ au désert vécu par les premiers ascètes du christianisme qui quittaient la société et se retiraient dans les déserts de Syrie et d'Egypte comme signe premier de leur rupture avec le monde. " L'ascète s'extrait donc de la société » (Kleinberg, 2005 : 158). L'ascète va au désert pour protester contre l'opulence de la société et se consacrer totalement à Dieu. Le désert était le lieu de ladite fuga mundi pour les virtuoses qui tendaient vers une " ascèse qui refuse le monde " (Weber, 1996: 194) Le désert est aujourd'hui plus difficile d'accès, d'une part parce qu'il se réduit et d'autre part parce que les ondes liées aux médias numériques ne laissent que peu de lieux où ces formes de communication ne sont pas disponibles. Même les monastères catholiques qui étaient à l'origine une forme institutionnalisée de fuite au désert sont de moins en moins en dehors du monde, autant par l'urbanisation accrue, que par l'arrivée de l'Internet et des réseaux de téléphonie mobile à l'intérieur même du monastère. En 2011, le monastère de la Pierrequi-Vire dans le Morvan, se réjouissait encore de se trouver dans un désert numérique sans accès au réseau de téléphonie mobile : «On est préservé du téléphone mobile, il n'y a pas de réseau. On considérait ça comme une bénédiction, je ne sais pas combien de temps ça va durer! On s'en réjouit! Beaucoup de gens sont addictifs au téléphone.» (04.2011) Entre le moment où j'ai commencé à étudier la vie monastique en 2004 et aujourd'hui, j'ai pu voir notamment l'évolution de la communication avec les moines qui passe de plus en plus par les courriers électroniques mais aussi l'arrivée de la connexion wifi dans les hôtelleries monastiques. Un trappiste français disait ainsi que la clôture, qui est censée les séparer du dehors, devient " plus poreuse ». Même le véritable désert n'est plus synonyme d'absence de réseau comme j'ai pu le voir lors d'une enquête au Kenya en 2014 avec de nombreuses publicités pour Safaricom, opérateur de téléphonie mobile qui dispose d'un réseau dans le désert du Nord Kenya. Le désert naturel ou social du monastère ne suffit donc plus pour se couper de la société. Il faut dans ce cas se couper volontairement et activement des systèmes de communication.

41 La déconnexion devient alors en soi le lieu de désert. Le désert ne sera vraiment désertique que s'il est affranchi des ondes magnétiques ou tout au moins des outils qui permettent de les recevoir. Ainsi, les jeûneurs créent leur désert en se déconnectant, comme l'explique par exemple un homme de 55 ans dans le questionnaire en allemand qui dit ne pas vouloir « d'influence de l'extérieur ». Une jeûneuse autrichienne qui fait chaque année seule des semaines de jeûne Buchinger-Lützner en prenant une semaine de vacances exprès, cherche un retrait total de la société et de ses actualités : 
« Je ne lis pas de journaux, je n'ai pas de télévision. Je ne l'allume jamais, s'il y en a une. Pendant ces cinq jours où je jeûne, je suis vraiment loin de ça. [...] De cette manière, je regarde aussi beaucoup, beaucoup moins la télévision depuis que je fais des jeûnes. C'est un long processus et je ne me laisse plus influencer par ça. [...] Je regarde mon téléphone seulement le soir. Pendant la journée, je l'éteins. S'il y avait quelque chose, si quelque chose se passait, je dis, ils doivent m'appeler et je rappelle. Mais pendant la journée, le téléphone est éteint, ça dérange sinon. Je dis à mes amis que je ne suis pas là, que personne n'appelle.» $(11.2015$, femme, 58 ans, employée de commerce)

Et elle ajoute plus loin : "J'entre vraiment en clôture ", reprenant une métaphore du monde monastique. En cela, la semaine de jeûne constitue une forme d'hétérotopie selon les termes de Foucault, un « contre-espace » qui se définit de manière différente par rapport aux espaces de la vie quotidienne. «Parmi tous ces lieux qui se distinguent les uns des autres, il y en a qui sont absolument différents : des lieux qui s'opposent à tous les autres, qui sont destinés en quelque sorte à les effacer, à les neutraliser ou à les purifier. Ce sont en quelque sorte des contre-espaces.» (Foucault, 2009: 24). Dans certains stages de déconnexion comme la semaine en Crête précédemment évoquée, une application est installée sur les smartphones des participants pour en bloquer toutes les fonctions hormis les appels.

\section{Se retrouver et trouver Dieu}

43 L'abstinence ou la réduction de l'utilisation des médias numériques apparaît tout d'abord pour les jeûneurs comme une condition pour atteindre un but général du jeûne qui serait celui de la subjectivité retrouvée, de «se retrouver». Ainsi, dans le questionnaire sur le jeûne, une jeune femme autrichienne de 28 ans explique : «Si le but est de 'se retrouver', se développer personnellement et pas simplement une raison corporelle, alors c'est logique que la consommation des médias ne soit pas vraiment appropriée.» Le jeûne des médias se rapporte dans ce cas à un "mode de subjectivisation spécifique » selon les termes de Foucault (Foucault, 1983).

La déconnexion renvoie alors à l'un des piliers de l'ascèse traditionnelle qui est le silence. Weber parle du "silence comme moyen de dompter l'ensemble de la vie personnelle » (Weber, 1996 : 151). Les Pères de l'Église insistaient sur cette ascèse qui ne doit pas concerner que la nourriture, mais toute pensée ou parole mauvaise. Jean Chrysostome exhorte : «Que non seulement la bouche jeûne, mais aussi l'œil, l'ouïe, les pieds, les mains et les autres membres du corps.» (cité par Noyé, 2007 : 117). Cette ascèse pour l'œil et l'ouïe ne peut être atteinte dans le monde actuel que par la déconnexion des technologies de la communication et de l'information. Celle-ci ne concerne pas uniquement les nouvelles technologies comme telles, mais tout média porteur d'informations constantes y compris les journaux pour limiter les interférences créées par le monde dans la confrontation avec soi-même. Le silence par l'absence de parole est aussi un élément présent dans les semaines de jeûne. Lors de la semaine de jeûne et randonnée à laquelle j'ai participé en Carinthie, le silence était de mise pendant toute la journée de marche. La responsable explique :

"C'est un exercice pour arriver au silence, parce que sinon, on ne peut pas entendre cette voix, cette voix intérieure. Je pense que les gens ne l'entendent plus parce qu'il y a tout simplement trop de bruit partout où on est. On est en permanence dans le bruit. [...] Et à travers le fait de ne pas parler et de faire silence, 
c'est tout simplement un exercice où l'on s'en rapproche, où l'on est inévitablement renvoyé à soi-même. » (06.2014, femme, 51 ans, coach de jeûne)

Le jeûne des médias numériques apparaît comme une véritable virtuosité puisqu'il se révèle souvent pour les jeûneurs plus difficile que l'abstinence alimentaire. Par exemple, une étudiante autrichienne de 24 ans qui a vécu deux semaines de jeûne Buchinger, explique : " J'ai jeûné de chocolat, de sucre et je fais maintenant le jeûne traditionnel de viande. J'ai aussi déjà essayé le jeûne de téléphone portable, mais je n'ai pas réussi. » Elle cite une autre amie qui aurait aussi essayé, mais n'aurait pas réussi. accueillir ce qui doit venir en soi, se débrancher du quotidien pour être plus dans une écoute profonde. » (Femme, 66 ans, retraitée)

«Aucun média pendant le jeûne, pour renouer avec soi.» (Femme, 49 ans, sans emploi)

« Faciliter l'intériorité. » (Femme, 75 ans, retraitée)

« Pour retrouver le silence intérieur indispensable à toute rencontre avec mon être profond. » (Femme, 58 ans, retraitée)

« Pour me recentrer, aller à l'écoute de mon intérieur plutôt qu'être assailli par le monde extérieur. » (Femme, 44 ans, intérimaire)

L'expérience des jeûneurs concerne bien ici « le développement des rapports à soi, pour la réflexion sur soi, la connaissance, l'examen, le déchiffrement de soi sur soi, les transformations qu'on cherche à opérer sur soi-même» (Foucault, 1983) qui correspond selon Foucault à l'«ascétique», entendue comme une forme de «subjectivisation » et « pratique de soi ».

Cet accès au soi est le passage incontournable qui ouvre sur la possibilité de l'expérience spirituelle de la transcendance. La réduction de la consommation des médias est pour de nombreux jeûneurs associée directement à une recharge plus intense en vie spirituelle. Des moines affirment que la réduction de leur usage d'Internet a directement pour but de prendre plus de temps pour Dieu. Certains jeûneurs associent l'abstinence des médias à une plus grande qualité de leur expérience spirituelle : "Pour être entièrement dans le jeûne et la prière " (Femme, retraitée), «Pour plus de spiritualité » (Femme, 69 ans, retraitée) ou " Pour me rendre disponible intérieurement à l'aventure spirituelle » (Femme, 65 ans, retraitée). Certains évoquent aussi directement la relation à Dieu, une plus grande intensification de cette relation qui serait permise par la déconnexion : «A fin d'une meilleure "connexion" avec Dieu et nous » (Femme, 52 ans, assistante administrative) ou "Pour se déconnecter et se relier autrement à Dieu» (Femme, 48 ans). La déconnexion technologique serait aujourd'hui la clef de la reconnexion aux petites et grandes transcendances.

\section{La nouvelle virtuosité}

Les virtuoses religieux se distinguent des masses selon Weber en cela qu'ils auraient " l'oreille musicale » pour les choses religieuses (Weber, 1996 : 362). La virtuosité ascétique renvoie à un mode de vie que les masses ne peuvent pas suivre parce qu'il serait inatteignable si l'individu ne possède pas le charisme de départ nécessaire.

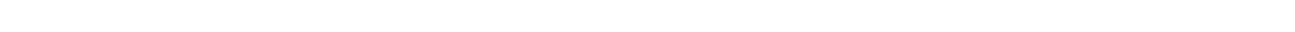


Fourquet-Courbet et Courbet, chercheurs en sciences de la communication, notent en effet une difficulté particulière qu'auraient les générations actuelles à s'abstenir des médias :

« En effet, parmi les multiples besoins et désirs que nous avons au quotidien (manger, boire, dormir, fumer, avoir des contacts sociaux, besoin d'hygiène, faire du sport...), le désir d'utiliser les médias (consulter ses e-mails, surfer sur le Web, aller sur les RSN, regarder la télévision) est celui pour lequel notre capacité de résister serait la plus faible. Non seulement le désir d'utiliser les médias serait plus fort et plus fréquent dans une journée que, par exemple, le désir de tabac, mais il serait, en outre, plus difficile à contrôler que les désirs de manger ou d'avoir des activités sexuelles. " (FouquetCourbet \& Courbet, 2017)

51 De même, le responsable du séminaire catholique du Graz, évoquant les semaines de jeûne Buchinger qu'il propose aux séminaristes comme retraite d'Avent, explique qu'il pense que c'est effectivement plus difficile de jeûner de téléphone portable que de nourriture :

« Je ne sais pas dans qu'elle mesure les séminaristes répondent honnêtement à cette question qu'ils ont reçue pendant la retraite. Mais je sais que ce n'est pas facile, concrètement pour beaucoup impossible, de ne pas utiliser leur téléphone pendant une semaine, et ne pas checker Whatsapp ou les SMS. C'est de fait impossible pour beaucoup, même s'ils le veulent. Mais justement, le fait d'en devenir conscient, c'est déjà quelque chose, de voir combien nous sommes dépendants de ces choses [...]» (06.2015, homme, 50 ans, supérieur du séminaire).

52 Même dans le cadre monastique, l'ascèse concernant les médias apparaît souvent plus difficile que d'autres domaines de la discipline monastique. Ainsi par exemple, un abbé autrichien reconnaissait qu'il est pour lui plus difficile de jeûner de séries sur Netflix que de viande. De même, les maîtres des novices affirment que l'abstinence totale de téléphone portable pendant la première année fait souvent partie des ascèses les plus difficiles pour les novices actuels.

\section{Une ascèse ostentatoire}

Le jeûne des médias numériques peut s'apparenter à une forme d'ascèse ostentatoire. Je définis comme ascèse ostentatoire une pratique ascétique qui se montre publiquement et qui tire une part de sa justification de l'étalage public. Selon Aviad Kleinberg, historien de l'ascèse chrétienne, "l'ascète opère sur trois plans parallèles à la fois : l'individuel, le culturel-idéologique et le social. » (Kleinberg, 2005 : 113). Le troisième plan de l'action sociale renvoie à la reconnaissance par la société. Kleinberg évoque même "le public » de l'ascète (Id : 116). Les stylites du IIIe siècle, comme Syméon Stylite, le plus connu, qui aurait passé trente ans de sa vie sur une colonne de plus de quinze mètres de haut où il ne pouvait même pas s'allonger, en sont un exemple. De nombreux curieux et pèlerins venaient lui rendre visite. Thorstein Veblen, dans son étude sur la consommation ostentatoire comme mode de consommation visant à indiquer un statut social, souligne comment les vêtements liturgiques du prêtre montre qu'il « ne se livre à aucun effort inutile » (Veblen, $1970: 120$ ). L'affichage sur les réseaux sociaux numériques du statut de jeûne ou l'annonce d'une semaine de retrait a autant un rôle pratique dans la communication de prévenir les interlocuteurs qu'un but ostentatoire pour signaler sa déconnexion et sa capacité à la tenir. Celle-ci peut alors jouer un rôle paradoxal de validation par les pairs dans le nombre de «j'aime » obtenu. 

congrégation sur l'impact des réseaux sociaux a annoncé se déconnecter pour 24 heures : «Je suis en 'sabbat numérique' pour $24 \mathrm{~h} . .$. Et si tu faisais l'expérience? ?. Elle a ensuite annoncé son « retour sur la toile » 24 heures plus tard, ce qui signifie que cette déconnexion d'une journée était véritablement considérée comme une épreuve. Un cistercien autrichien a quant à lui obtenu 231 «j'aime » en publiant en septembre 2018 qu'il allait être hors-ligne pour quelques jours. Le but pratique est de prévenir le réseau que l'on ne sera pas joignable et que l'on ne répondra pas pour éviter des incompréhensions, ce qui, dans un sens, réduit la virtuosité de la démarche. Comme évoqué plus haut, ce type de jeûne prend place dans un contexte où l'usage des médias numériques n'est plus une forme de distinction en soi. Elisabeth Castro-Thomasset avait remarqué concernant la télévision que « rejeter un objet que tout le monde possède, c'est-à-dire qui s'inscrit dans une pratique sociale normative, signifie qu'en optant pour la rupture, on tente d'échapper à la pression sociale d'une attitude conformiste » (Castro-Thomasset, $2000: 21$ ). Ce que Thilo von Pape et Corinne Martin (2010: 125) reprennent pour le téléphone portable. Ainsi le choix d'abstinence est justifié et mis en scène sur les réseaux sociaux en ligne. «Il s'agit ainsi non seulement d'une manière de raconter sa vie et d'intéresser les autres, mais aussi de gérer l'image qu'il ou elle donne de soi. » (Casilli, 2012: 19). Et c'est finalement la déconnexion qui devient un élément de la distinction.

\section{Conclusion}

Le jeûne des médias numériques, intégré à un jeûne alimentaire à portée spirituelle ou prenant place dans un contexte de jeûne religieux institutionnel, est directement mis en lien par les acteurs avec une recharge en spiritualité. En cela, le jeûne d'Internet semble remplir un rôle similaire au jeûne alimentaire dans la libération d'influences extérieures, la maîtrise des désirs pour augmenter la concentration sur soi et sur Dieu. À partir du moment où l'acteur s'abstient volontairement d'Internet dans un but spirituel, cela signifie qu'il intègre cette consommation à son comportement religieux de manière générale comme cela peut être le cas du comportement sexuel par exemple. Il s'agit donc bien d'une ascèse. Dans le contexte actuel où les ondes laissent peu de lieux épargnés, le renoncement partiel ou total aux médias numériques apparaît en effet comme un élément récurrent des pratiques de jeûne. Le nouveau désert recherché traditionnellement par les ascètes serait aussi celui de la déconnexion. Toutefois, le paradoxe de cette ascèse est qu'elle se fait aussi ostentatoire en se mettant en scène dans les lieux auxquels elle dit renoncer.

Du côté de l'Eglise institutionnelle, le jeûne des médias pendant le carême ou l'avent est un moyen de remettre l'idée d'abstinence pendant un temps déterminé au goût du jour alors que le jeûne alimentaire de ces périodes, comme prescription de l'Eglise, est rarement suivi. Ainsi par exemple la proposition «freestyle Fasten» (jeûne en freestyle) de la pastorale des jeunes autrichienne qui se positionne comme forme de "subjective-live " pour reprendre les termes de Heelas et Woodhead (2005) par l'idée de liberté de la démarche est une façon d'intégrer le courant de jeûne des médias numérique pour tenter de regagner en plausibilité.

57 J'ai évoqué ici les pratiques de déconnexion comme jeûne en soi ou dans le cadre d'un jeûne alimentaire à but spirituel. Mais il faut aussi évoquer le cas où des jeûneurs qui

RESET, 9 | 2020 
effectuent leur session de jeûne alimentaire seuls vont chercher le soutien d'un groupe par le biais des réseaux sociaux numériques. Dans ce cas, la déconnexion n'est pas à l'ordre du jour car les réseaux sociaux en ligne jouent le rôle de communauté recherchée dans l'expérience liminale (Turner, 1969: 360) De même, certains catholiques pratiquants vont avoir particulièrement recours aux offres religieuses en ligne lors de moments de réintensitication de leur vie spirituelle. Ainsi dans le questionnaire diffusé par le biais des offres spirituelles en ligne des franciscains autrichiens, 17,3\% des répondants disent augmenter leur consommation des médias numériques dans un but religieux lors des moments importants pour la foi comme le carême.

\section{BIBLIOGRAPHIE}

ASSOULY Olivier (2002). Les Nourritures divines, Essai sur les interdits alimentaires, Paris, Actes Sud, 2002.

CASILLI Antonio (2012). «Etre présent en ligne : Culture et structure des réseaux sociaux d'Internet », Idées économiques et sociales, 3/169, pp.16-29.

CASTRO-THOMASSET Elisabeth (2000). L'apostasie de la télévision. Une forme d'iconoclasme contemporain, Paris, L'Harmattan.

ČEJKA Marek (2009). « Making the Internet Kosher: Orthodox (HAREDI) Jews and their Approach to the World Wide Web », Masaryk University Journal of Law and Technology, pp.1-13.

COHEN Yoel (2013). « Awkward encounters. Orthodox Jewry and the Internet », in Tore Ahlbäck (dir.), Digital Religion, Turku, Donner Institute for Research, pp.42-54.

FOUCAUlT Michel (1983). « Usage des plaisirs et techniques de soi », Le Débat, 27, pp. 46-72. [En ligne : http://1libertaire.free.fr/MFoucault177.html]

FOUCAULT Michel (1994). Dits et écrits 1954-1988, Paris, Gallimard.

FouCAult Michel (2009). Le Corps utopique, Les hétérotopies, Paris, Editions Lignes.

FOURQUET-COURBET Marie-Pierre, COURBET, Didier (2017). « Anxiété, dépression et addiction liées à la communication numérique. Quand Internet, smartphone et réseaux sociaux font un malheur ", Revue Française des Sciences de l'Information et de la Communication, [En ligne], mis en ligne le 01 août 2017, consulté le 25.09.2017. URL : http://rfsic.revues.org/2910.

GRANJON Fabien (2010). « Le "non-usage” de l'internet : reconnaissance, mépris et idéologie », Questions de communication, $\mathrm{n}^{\circ} 18, \mathrm{pp} .37-62$.

HEELAS Paul, WOODHEAD Linda (2005). The Spiritual Revolution. Why Religion is giving way to spirituality?, Oxford, Blackwell.

HELLAND Christopher (2005). « Online Religion as Lived Religion: Methodological Issues in the Study of Religious Participation on the Internet », Online-Heidelberg Journal of Religions on the Internet 1(1). [En ligne] 
HERVIEU-LÉGER Danièle (1993). La religion pour mémoire, Paris, Le Cerf.

HÖLLINGER Franz, TRIPOLD Thomas (2012). Ganzheitliches Leben. Das holistische Milieu zwischen neuer Spiritualität und postmoderner Wellness-Kultur, Bielefeld, Transcript.

JONVEAUX Isabelle (2013). Dieu en ligne. Expériences et pratiques religieuses sur Internet. Paris, Bayard. JONVEAUX Isabelle (2018). Mönch sein heute. Eine Soziologie des Mönchtums in Österreich im europäischen Dialog. Wurtzbourg, Echter.

KELLNER Catherine, MASSOU Luc, MORELLI Pierre (2010). «(Re)penser le non-usage des tic », Questions de communication, $\mathrm{n}^{\circ} 18$, pp. 7-20.

KLEINBERG Aviad (2005). Histoires de saints. Leur rôle dans la formation de l'Occident (trad. Moshé Méron), Paris, Gallimard.

KNOBLAUCH Hubert (2009). Populäre Religion. Auf dem Weg in eine spirituelle Gesellschaft, Francfort-surle-Main, Campus Verlag.

LE GUEN-FORMENTI Lucie (2015). 'De la mise en scène pudique comme expression d'une morale musulmane sur Youtube', Tic\&Société, 9(1-2), En ligne : https://journals.openedition.org/ ticetsociete/1872.

LIVINGSTONE Sonia, HELSPER Ellen (2007). « Gradations in digital inclusion : children, young people and the digital divide », New Media and Society, 9 (4), pp. 671-696.

NOYÉ Jean-Claude (2007). Le grand livre du jeûne, Paris, Albin Michel, 2007.

SHORT Mary, KASPER, Thomas, WETTERNECK, Chad (2015). « The relationship between religiosity and internet pornography use », Journal of Religious Health, 54(2), pp.571-583.

TURNER Victor (1969). « Liminality and Communitas », in The Ritual Process: Structure and AntiStructure, Chicago, Aldine Publishing.

VEBLEN Thorstein (1970). Théorie de la classe de loisir (trad. Evrard Louis), Paris, Gallimard. VON PAPE Thilo, MARTIN Corinne (2010). « Non-usages du téléphone portable. Au-delà d'une opposition binaire usagers/non-usagers », Questions de communication, 18, pp. 113-143.

WEBER Max (1996). Sociologie des religions, Paris, Gallimard.

WEBER Max (2002). L'éthique protestante et l'esprit du capitalisme (trad. Kalinowski Isabelle), Manchecourt, Flammarion.

\section{NOTES}

1. Financée par le FWF Der Wissenschaftsfonds projet M 1271-G15 «Asceticism in modern monasticism : changing religious body ». Publication Moines corps et âme (2018).

2. Projet „Aspiration to the simple life“, finance par le FWF (Autriche), Projet Elise-Richter V304V15, 08.2013-10.2017.

3. Posté en novembre 2016 dans le groupe fermé « Fasten/Heilfasten », il a récolté 65 réponses.

4. Ce questionnaire envoyé en février 2017 a récolté 83 réponses. Il a été envoyé par la secrétaire de l'association aux anciens participants des sessions.

5. https://www.youbeee.com/Angebot/digital+detox+retreat+auf+kretaABVD364011/10.06.2017-17.06.2017/1501 [consulté le 13.12.2016].

6. Le Baromètre du numérique, CREDOC, 2016, p.178. 
7. «Exodus 90 » se veut un programme d'exercices spirituels ascétiques «pour hommes catholiques » originellement élaboré à destination de séminaristes aux Etats-Unis. Un entretien a été mené avec un participant de ce programme.

\section{RÉSUMÉS}

L'utilisation des médias numériques pour la pratique religieuse ne fait plus figure d'exception et semble au contraire être devenue pratique courante. Mais parallèlement, apparaissent de plus en plus de démarches de renoncement total ou partiel à l'utilisation d'Internet pendant une période particulière de réintensification de la vie religieuse ou spirituelle. Prenant l'exemple de l'ascèse des virtuoses religieux avec les moines et moniales catholiques, du carême chrétien et des stages de jeûne et randonnée qui s'inscrivent dans une approche de spiritualité globale, cet article montre grâce à des données de terrain comment les acteurs réduisent consciemment leur consommation d'Internet à des fins religieuses. Certains parlent même d'un jeûne d'Internet qui serait le pendant indispensable du jeûne alimentaire. Toutefois, dans de nombreux cas, la déconnexion apparaît plus difficile que le jeûne alimentaire et s'affiche alors comme un nouveau type de virtuosité. Réexaminant les catégories classiques pour la sociologie des religions que sont le jeûne et l'ascèse, cet article montre comment elles se redéfinissent aujourd'hui dans leurs nouveaux objets d'application. Pour l'Eglise institutionnelle, c'est aussi l'occasion de redonner une plausibilité à des pratiques - notamment le jeûne du carême - qui étaient peu à peu tombées en désuétude.

The use of digital media for the religious practice is no longer the exception, but seems to have become a common practice. Parallel to this, we observe more and more forms of total or partial renouncement of use of the Internet during a particular period of reintensification of the religious or spiritual life. Taking the examples of asceticism of religious virtuosi with Catholic monks and nuns, Christian Lent and fasting and hiking weeks that are part of a holistic spirituality approach, this article shows with the help of empiric data how actors consciously reduce their use of the Internet for religious purposes. Some even speak of an Internet fast which would be an indispensable counterpart to the food fast. However, in many cases, disconnection appears to be more difficult than food fasting and is then seen as a new type of virtuosity. Reexamining the classical categories of the sociology of religions that are fasting and asceticism, this article shows how they are redefined today with new objects of application. For the institutional Church, it is also an opportunity to restore plausibility to practices - especially the Lenten fast - which had gradually fallen into disuse.

\section{INDEX}

Keywords : Internet, fast, non-use, Catholicism, monastery, holistic spirituality

Mots-clés : internet, jeûne, non-usage, ascèse, catholicisme, monastère, spiritualité globale 
AUTEUR

ISABELLE JONVEAUX

Université de Graz, CéSor 